\title{
Análisis termodinámico de un disco de freno automotriz con pilares de ventilación tipo NACA 66-209
}

\section{Thermodynamic analysis of an automotive brake disc with ventilation pillars NACA type 66-209}

DOI: https://doi.org/10.17981/ingecuc.14.2.2018.01

Artículo de investigación. Fecha de recepción: 12/01/2018. Fecha de aceptación:.21/06/2018

\author{
Ricardo Andrés García-León \\ Universidad Francisco de Paula Santander. Ocaña (Colombia). \\ ragarcial@ufpso.com \\ Robert Dajjan Echavez-Díaz \\ Universidad Francisco de Paula Santander. Ocaña (Colombia). \\ rdechavezd@ufpso.com \\ Eder Flórez-Solano \\ Universidad Francisco de Paula Santander. Ocaña (Colombia). \\ enflorezs@ufpso.com
}

Para citar este artículo:

R. A. García-León, R. D. Echavez-Díaz y E. Flórez-Solano, "Análisis termodinámico de un disco de freno automotriz con pilares de ventilación tipo NACA 66-209," INGE CUC, vol. 14, no. 2, pp.9-18, 2018. DOI: http://doi.org/10.17981/ingecuc.14.2.2018.01

\section{Resumen}

Introducción- El sistema de frenado de un automóvil debe trabajar de forma segura y predecible en cualquier circunstancia, lo cual implica disponer de un nivel estable de fricción en cualquier condición de temperatura, humedad y salinidad del medio ambiente. Para un correcto diseño y operación de los discos de freno, es necesario considerar diferentes aspectos, tales como: la geometría, el tipo de material, la resistencia mecánica, la temperatura máxima, la deformación térmica, la resistencia al agrietamiento, entre otros.

Objetivo- En el presente trabajo se realizó el análisis del sistema de freno a partir del pedal como inicio de los cálculos de cinética y dinámica de los elementos constitutivos, $\mathrm{y}$, de esta manera, simular el comportamiento de un freno automotriz con pilares de ventilación tipo NACA 66-209.

Metodología- El desarrollo de la investigación se llevó a cabo mediante la ejecución de un Análisis de Elementos Finitos (FEA) con la ayuda del programa de computador SolidWorks Simulation, con el que se llevó a cabo el modelo geométrico del disco para identificar los elementos sometidos a máximas variaciones de temperatura.

Resultados- Con los resultados numéricos obtenidos se demuestra que, con los cálculos matemáticos, se logró validar el correcto funcionamiento de sistema de frenado en diferentes condiciones de operación, optimizando el tipo de geometría de los discos y ayudando así a la evacuación más rápida de calor con respecto a otros tipos de frenos de disco.

Conclusiones- Estos sistemas trabajan en condiciones óptimas, es decir, velocidad de $80 \mathrm{Km} / \mathrm{h}$ y en un medio ambiente de $22{ }^{\circ} \mathrm{C}$, generando una temperatura de frenado de $60,5^{\circ} \mathrm{C}$. Estos valores garantizan altos niveles de seguridad y operación en comparación con otros tipos de geometrías, además de poder determinar sus condiciones de funcionamiento en diferentes condiciones de trabajo.

Palabras clave- Termodinámica; fricción; FEA; CFD; frenos de disco, automóvil; NACA 66-209

\section{Abstract}

Introduction- The braking system of a car must work safely and predictably under any circumstance. This implies having a stable level of friction in any condition of temperature, humidity and salinity of the environment. For a correct design and operation of the brake discs, it is necessary to consider different aspects, such as geometry, type of material, mechanical resistance, maximum temperature, thermal deformation, resistance to cracking, among others.

Objective- In the present work, the analysis of the brake system from the pedal was carried out as the beginning of the kinetic and dynamic calculations of the constituent elements and, in this way, simulate the behavior of an automotive brake with NACA 66-209.

Methodology- The development of the investigation was carried out by means of the execution of a Finite Element Analysis (FEA) with the help of the SolidWorks Simulation Software. The geometric model of the disk was carried out in order to identify the elements submitted to maximum temperature variations.

Results- The results correspond to the numerical results of the tests that were carried out, in this case, optimizing the disc geometry type so as to aid in a faster heat evacuation of disc brakes.

Conclusions- These systems work in optimal conditions, that is to say: speed of $80 \mathrm{Km} / \mathrm{h}$ and in an environment of $22^{\circ} \mathrm{C}$, generating a braking temperature of $60.5{ }^{\circ} \mathrm{C}$. These values guarantee high levels of safety and operation compared to other geometry types. In addition, operating conditions under different working conditions can be determined.

Keywords- Thermodynamics; friction; FEA; CFD; disc brakes; automobile; NACA 66-209 


\section{INTRODUCCIÓN}

El sistema de frenos es, sin duda, el componente más importante para la seguridad vial del automóvil, ya que de este depende la detención total o parcial del vehículo, y, en consecuencia, la integridad de sus pasajeros. Generalmente, el $70 \%$ de la energía cinética producida en el movimiento es absorbida por los frenos de disco delanteros y el restante por el freno trasero, que suele ser de tambor. Estos sistemas se fundamentan en la fricción para detener el movimiento del vehículo, teniendo como principio la presión hidráulica que empuja las pastillas de freno contra el disco de hierro fundido. En consecuencia, el comportamiento que produce este tipo de dispositivos, a través de la energía cinética, es el de crear un calor considerablemente alto durante el frenado, aumentando la temperatura por fricción. Este calor se disipa rápidamente con el aire circundante por medio del fenómeno de convección (transferencia de calor que se produce entre masas a diferente temperatura), y esto depende de la geometría del disco y del material del que se fabrica. Los factores ambientales también son determinantes para que la etapa de transferencia de calor se produzca. Además, cuando la temperatura alcanza valores elevados aparece el fenómeno de radiación, que también ayuda a disipar la energía en forma de calor almacenada en el disco [1][2][3]. Bajo estas condiciones, la funcionalidad y la seguridad del freno del sistema pueden estar comprometidas.

La determinación de las características geométricas de los discos depende de la capacidad de carga y el funcionamiento, lo cual es un factor importante en la fase de diseño inicial. En la mayoría de los casos, el diseño de los discos debe evitar el sobrecalentamiento que surge entre el freno y la pastilla por el efecto de la fricción, seleccionando de forma adecuada las propiedades físicas, mecánicas y químicas que se producen debido a que en algunas ocasiones los tipos de materiales no se comportan correctamente y tienen efectos negativos sobre la eficacia del proceso de frenado [4]. En la etapa del diseño mecánico en frenos de disco ventilados, es muy importante analizar el comportamiento de los termo-fluidos asociados (aire circundante), es decir, observar las características y operación de los fluidos sobre la superficie del disco, siempre garantizando la efectividad del proceso de frenado y de disipación de calor por la superficie y los canales de ventilación [5]. El calor de disipación térmica y el rendimiento de los discos de frenos ventilados depende en gran medida de las características del flujo de aire a través de los canales de ventilación y las configuraciones de geometría de los discos de freno, los cuales se comprueban mediante la implementación de programas de diseño que posean la biblioteca de la Dinámica de Fluidos Computacionales "CFD", como es el caso de SolidWorks Simulation [6][7][8][9][10].
En general, las aplicaciones de CFD en la industria del automóvil han recorrido un largo camino para influir en el diseño de componentes de automoción debido a los continuos avances en hardware y software, así como los avances en las técnicas numéricas para resolver las ecuaciones de flujo de fluidos. El interés de la industria del automóvil en aplicaciones CFD (Dinámica de Fluidos Computacional) se deriva de su capacidad para mejorar el diseño de automóviles y para reducir el costo del producto y el tiempo de ciclo de vida de los productos [11][12][13]. Por lo anterior, el estudio de los efectos dinámicos de los discos de freno es un área importante de la investigación para los fabricantes de la industria, así como para el mundo académico.

Actualmente, investigadores de la University of Technology Sydney simularon, en un banco de pruebas, el comportamiento del disco de freno para obtener el beneficio económico y dinámico en el rendimiento del sistema, con lo que se comprobó la eficiencia del sistema en diferentes condiciones de operación [14][15]. Asimismo, se obtuvieron perfiles de frenado mediante el modelado y análisis numéricos de los componentes del sistema con la finalidad de predecir su comportamiento en diferentes usos a los que puede ser sometido, teniendo en cuenta los resultados del análisis de elementos finitos (FEA) para validar los resultados [16]. Además, en [17] se estudió el efecto de la dinámica longitudinal en trenes que funcionaban con frenos de aire prediciendo las características del sistema.

En 2015, científicos utilizaron el método de elementos finitos con la ayuda del programa CATIA para predecir fallas en el disco de freno y, de esta manera, identificar las zonas más críticas, teniendo en cuenta que estos sistemas están fabricados de fundición gris nodular, obteniendo resultados importantes en la optimización de los sistemas de frenado [18][19][20].

En la investigación realizada por [21], consideraron como componente principal del sistema de frenos al pedal con el fin de estudiar las condiciones en cálculos dinámicos. Se obtuvieron resultados matemáticos en condiciones estáticas y dinámicas del sistema para predecir su comportamiento. La fuerza del pedal (PF), el recorrido del pedal (PT) y el tiempo de respuesta (RT) son estudiados con la finalidad de satisfacer las expectativas de seguridad mediante análisis estáticos y dinámicos en diferentes tipos de automotores y en diferentes condiciones de trabajo, resultó de gran ayuda aplicar estas herramientas para investigaciones en ingeniería [22]. Además, se ha analizado la influencia de las cargas en el sistema de frenado y de la fuerza en el pedal del freno debido a que influyen directamente en la dinámica natural de los componentes [23].

Con la finalidad de cumplir los requisitos de frenado y optimizar los recursos tecnológicos de los sistemas de freno, investigadores propusieron un frenado 
de alta frecuencia utilizando un accionador magnético, para así resolver los problemas del frenado hidráulico. Los resultados se analizaron con la ayuda del programa LabVIEW y por medio del análisis de elementos finitos FEA [24].

En la actualidad se utilizan frenos de disco ventilados debido a que poseen altas características de disipación de calor. Investigaciones recientes han demostrado que los discos ventilados tienen altas velocidades de transferencia de calor debido a un aumento en la turbulencia, que se traduce en un mayor coeficiente de transferencia calor. Los discos ventilados también tienen una mayor resistencia a la deformación térmica debido a la distribución uniforme del material, lo que reduce el estrés térmico acumulado dentro del rotor, que depende principalmente de la geometría del disco y de la configuración óptima de los canales de ventilación [25][26]. Además, el mantenimiento de los frenos de disco es más económico en comparación con los frenos de tambor [27].

\section{Materiales y Métodos}

La energía cinética y potencial del vehículo se transforma rápidamente en energía térmica por los frenos cuando se realizan frenados repetitivos. Para que el sistema de frenado funcione correctamente, el calor generado debe disiparse lo más rápidamente posible para que los frenos sucesivos no sobrecalienten el sistema y comprometan así el rendimiento y la seguridad. El movimiento del vehículo permite la disipación del calor por convección y radiación, comportamientos típicos en transferencia de calor. Un frenado intenso hace que la temperatura aumente hasta cierto límite, conocida como la temperatura de saturación, que depende de la capacidad de disipación térmica del disco de freno. Se obtuvieron las propiedades físicas y térmicas de los discos. Se analizaron los materiales del disco y se encontró que los discos consistían en hierro fundido gris nodular con grafito laminar, silicio y manganeso. Las propiedades físicas y térmicas de este material fueron determinadas teniendo en cuenta las referencias de Cengel y Riley [28][29].

El Comité Asesor Nacional para la Aerodinámica (NACA), predecesor de la NASA, es el organismo que se encarga del diseño de los perfiles más eficientes según las diferentes necesidades que surgen en la aviación mundial desde los años de 1930. Los perfiles fueron generados usando ecuaciones analíticas que describen la curvatura de la línea central geométrica o la línea de curvatura media, así como la distribución del espesor a lo largo de la longitud del perfil [30].

Con la finalidad de llevar a cabo el proceso metodológico, se seleccionó el perfil para el desarrollo de la investigación, que fue el NACA 66-209 (Fig. 1), correspondiente a la serie 6 , con un rango de máximo espesor del $9 \%$ al $45 \%$ de la longitud de la cuerda y un rango de máxima curvatura de entre el $1.1 \%$ y el $50 \%$ de la cuerda, con un coeficiente de sustentación de 0,2 y la ubicación del punto de mínima presión en el $60 \%$ de la longitud de la cuerda [31][32].

Teniendo en cuenta lo anterior, el perfil tipo NACA 66-209 minimiza el coeficiente de resistencia, comúnmente llamada serie Airfoil, el cual tiene como objetivo minimizar la resistencia asegurando un flujo laminar constante, reduciendo el gradiente de presión adversa, además de aumentar las características de máxima sustentación. La nomenclatura de este tipo de perfiles consta de 5 dígitos, siendo siempre el primero el número 6 , el cual hace referencia a la serie. El segundo dígito corresponde a la ubicación del punto de mínima presión en décimas de la cuerda, medidas desde el borde de ataque. El tercer número indica el coeficiente de sustentación de diseño y, finalmente, los dos últimos valores otorgan el máximo grosor en porcentaje de la cuerda. Existen otras modificaciones y variaciones de los perfiles de álabes pero son relativos a la función con que se quieran operar.

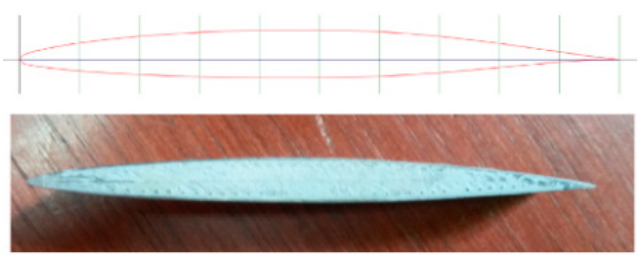

Fig. 1. Perfil NACA 66-209.

Fuente: elaboración propia.

Habiendo seleccionado el tipo de geometría de los álabes, se procedió a diseñar un freno de disco con pilares de ventilación tipo Naca. Teniendo en cuenta investigaciones realizadas por [33] y con la ayuda de libros de transferencia de calor, se llevaron a cabo las siguientes características en los cálculos matemáticos:

\section{A. Cálculo dinámico del sistema de frenado}

Energía disipada por el sistema de frenos. El frenado se produce gracias al rozamiento entre las pastillas y los discos de freno, transformando la energía cinética del vehículo en calor. Para calcular esta energía disipada es necesario saber la masa del vehículo, el factor de corrección $i$ para masas rodantes y la velocidad que lleva el vehículo al momento que empieza a frenar. Para un vehículo convencional su masa $m$ es aproximadamente de $1930 \mathrm{Kg}$, el factor de corrección para masas rodantes $i$ para un vehículo de velocidad media es 1,05, basados en [34], y tomando una velocidad de $80 \mathrm{Km} / \mathrm{h}$ para efectos de cálculo (equivalente a $22,22 \mathrm{~m} / \mathrm{s}$ ) se obtiene el siguiente valor de energía disipada en el freno trasero:

$$
\mathrm{E}_{\mathrm{f}}=\frac{\mathrm{i} * \mathrm{~m} *\left(\mathrm{~V}_{\mathrm{i}}^{2}-\mathrm{V}_{\mathrm{f}}^{2}\right)}{2}=500270,3 \mathrm{~J}
$$


Para calcular la energía disipada en el freno delantero $\left(\mathrm{E}_{\mathrm{d}}\right)$ es necesario saber que la energía total se distribuye entre un $75 \%$ y $80 \%$ en el eje delantero debido a que la mayor cantidad de masa del vehículo se presenta en ese eje, de lo que resulta:

$$
\mathrm{E}_{\mathrm{d}}=\mathrm{E}_{\mathrm{f}} * 0,75=375202,72 \mathrm{~J}
$$

Del total de energía que se absorbe en el eje delantero, un $90 \%$ se disipa en el disco de freno y un $10 \%$ en la pastilla, como lo indica [35].

$$
\mathrm{E}_{\text {disco }}=\mathrm{E}_{\mathrm{d}} * 0,9=337682,45 \mathrm{~J}
$$

Un automóvil tiene 2 ejes y cada eje tiene dos discos, así que la energía disipada se reparte entre ellos:

$$
\mathrm{E}=\frac{\mathrm{E}_{\mathrm{disc}}}{2}=168841,22 \mathrm{~J}
$$

La optimización del sistema de frenado en este caso es que la energía se disipe en el menor tiempo posible y, de esta manera, evitar sobrecalentamientos y fallas del sistema.

Fuerza de roce sobre las pastillas. Para calcular la fuerza de roce en la pastilla es necesario tener un factor de adherencia entre la calzada y el neumático. Para este caso se toma un factor de 0,93 , teniendo en cuenta las referencias en condiciones de una calzada seca [36].

La tabla 1 muestra los coeficientes de adherencia $\mu$ de neumáticos sobre calzadas en diferente estado, con distintos estados de los neumáticos y a diferentes velocidades.

Tabla 1. Coeficientes de Adherencia.

\begin{tabular}{|c|c|c|c|}
\hline $\begin{array}{c}\text { Velocidad } \\
\text { de Marcha } \\
\mathbf{( K m} / \mathbf{H})\end{array}$ & $\begin{array}{c}\text { Estado de los } \\
\text { Neumáticos }\end{array}$ & $\begin{array}{c}\text { Carretera } \\
\text { Seca (M) }\end{array}$ & $\begin{array}{c}\text { Carretera } \\
\text { Mojada (M) }\end{array}$ \\
\hline \multirow{2}{*}{50} & Nuevo & 0,85 & 0,65 \\
\cline { 2 - 4 } & Usado & 1 & 0,5 \\
\hline \multirow{2}{*}{80} & Nuevo & 0,8 & 0,6 \\
\cline { 2 - 4 } & Usado & 0,95 & 0,2 \\
\hline \multirow{2}{*}{120} & Nuevo & 0,75 & 0,55 \\
\cline { 2 - 4 } & Usado & 0,9 & 0,2 \\
\hline
\end{tabular}

Fuente: [1]

Por lo tanto, resulta que:

$\mathrm{F}_{\mathrm{r}}=\mathrm{u}_{\mathrm{b}} * \mathrm{~F}_{\mathrm{n}}$

Donde $\mathrm{u}_{\mathrm{b}}$ es el coeficiente de adherencia y Fn es la fuerza normal al vehículo; reemplazando se obtiene:

$$
\begin{aligned}
& \mathrm{F}_{\mathrm{n}}=1930 \mathrm{~kg} * 9,81 \mathrm{~m} / \mathrm{s}=18.933,3 \mathrm{~N} \\
& \mathrm{~F}_{\mathrm{r}}=0,93 * 18.933,3 \mathrm{~N}=17.607,96 \mathrm{~N}
\end{aligned}
$$

Ésta es la fuerza necesaria para que la fricción entre la pastilla y el freno pueda desacelerar por completo el vehículo en una calzada seca.
Longitud de frenado. Para determinar la longitud de frenado es necesario conocer la masa del vehículo, la fuerza de roce sobre las pastillas y la velocidad a la que el vehículo comienza a frenar. Para estos cálculos la velocidad es de $80 \mathrm{Km} / \mathrm{h}=22,22 \mathrm{~m} / \mathrm{s}$.

$$
\mathrm{l}=\frac{1}{2} * \frac{\mathrm{m}}{\mathrm{g} * \mathrm{~F}_{\mathrm{r}}} *\left(\mathrm{~V}_{\mathrm{i}}^{2}-\mathrm{V}_{\mathrm{f}}^{2}\right)=27,05 \mathrm{~m}
$$

Tiempo de frenado. Este tiempo es necesario para ver si un disco de freno es óptimo en relación con otros tipos, ya que la integridad del conductor se ve involucrada en este lapso de tiempo. Para calcularlo se necesita la distancia de frenado y la velocidad a la que el vehículo empieza a frenar.

$$
\mathrm{t}=\frac{2\left(\mathrm{x}_{\mathrm{f}}-\mathrm{x}_{\mathrm{i}}\right)}{\left(\mathrm{v}_{\mathrm{f}}-\mathrm{v}_{\mathrm{i}}\right)}=2,43 \mathrm{~s}
$$

Cálculo del calor generado en el proceso de frenado. Para determinar la temperatura que se genera en el proceso de frenado, es necesario tener en consideración el área de la pastilla de freno, el tiempo que se demora en detenerse el vehículo, la longitud de la pastilla y las propiedades térmicas y físicas de los elementos de freno.

Se debe tener la potencia por unidad de área para el cálculo del calor generado. Para esto es necesario el valor del área de la pastilla, que es donde se produce la fricción, el tiempo de frenado y la energía disipada en el disco de freno.

Elárea de una pastilla convencional es de 29.996,2 $\mathrm{mm}^{2}[36]$.

$$
q_{\text {total }}=\frac{E}{a * t}=2.316 .359,52 \mathrm{~W} / \mathrm{m}^{2}
$$

Ésta es la potencia por unidad de área que se produce entre el disco y la pastilla en el freno.

\section{B. Análisis térmico del disco de freno.}

La potencia disipada en el sistema de frenos que proviene de la energía cinética y potencial del vehículo se transforma en energía calorífica, la cual va a ser generada en un corto intervalo de tiempo. Para que el sistema de frenos funcione correctamente, el calor generado debe ser evacuado con la mayor rapidez posible, para que las sucesivas frenadas no produzcan una elevada temperatura y así perder la seguridad del sistema. El propio avance del vehículo permite la disipación del calor generado esencialmente por convección y radiación. Cuando se producen frenadas excesivas, la temperatura se eleva hasta llegar a un límite (temperatura de saturación) en función de la capacidad de disipación térmica del disco de freno [1].

Las propiedades físicas y térmicas de la fundición gris nodular de grafito laminar, el cual tiene una composición de silicio y manganeso, fueron obtenidas de la Tabla A-3 del libro de transferencia de calor de Yunus A. Cengel [37]: 
Conductividad térmica: $\mathrm{k}=41 \mathrm{~J} / \mathrm{s} * \mathrm{~m} *{ }^{\circ} \mathrm{C}$

Calor específico: $\mathrm{C}_{\mathrm{p}}=434 \mathrm{~J} / \mathrm{Kg} *{ }^{\circ} \mathrm{C}$

Densidad: $\rho=8131 \mathrm{Kg} / \mathrm{m} 3$

Difusividad térmica: $a=11,6 * 106 \mathrm{~m}^{2} / \mathrm{s}$

Coeficiente de transmisión térmica:

$\mathrm{U}=32 \mathrm{~J} / \mathrm{s} * \mathrm{~m}^{2} *{ }^{\circ} \mathrm{C}$

La elevación de temperatura del conjunto de un freno se evalúa por la siguiente expresión:

$$
\Delta \mathrm{T}=\frac{\mathrm{E}_{\mathrm{disc}}}{\mathrm{m} * \mathrm{C}_{\mathrm{p}}}=38,5^{\circ} \mathrm{C}
$$

Para calcular la temperatura en la superficie del disco se utiliza la siguiente ecuación:

$$
\mathrm{T}_{1}-\mathrm{T}_{\infty}=\Delta \mathrm{T}
$$

Donde $\mathrm{T}_{\infty}$ es la temperatura ambiente de $22^{\circ} \mathrm{C}$, entonces la temperatura en la superficie del disco es

$$
\mathrm{T}_{1}=60,5^{\circ} \mathrm{C}
$$

Lo que nos indica que, para un vehículo con una masa de $1930 \mathrm{Kg}$ que lleva una velocidad de $80 \mathrm{Km} / \mathrm{h}$ y circula en un ambiente de $22^{\circ} \mathrm{C}$, la temperatura que se genera en el disco de freno hasta detenerse es de aproximadamente $60,5^{\circ} \mathrm{C}$.

Enfriamiento de Newton. Un objeto sometido a una temperatura T1 se enfriará si se deja a temperatura ambiente, de acuerdo con la ecuación exponencial [37]:

$$
\mathrm{T}_{\mathrm{i}}-\mathrm{T}_{\infty}=\left(\mathrm{T}_{1}-\mathrm{T}_{\infty}\right) * \mathrm{e}^{-\frac{\mathrm{A}^{*} \mathrm{U}}{\mathrm{m} \text { Cp }} * \mathrm{t}}
$$

Donde:

$\mathrm{T}_{\mathrm{i}}=$ Temperatura instantánea en cada momento en ${ }^{\circ} \mathrm{C}$

$\mathrm{A}=$ Área de transferencia de calor en $\mathrm{m}^{2}$

$\mathrm{U}=$ Coeficiente de transmisión térmica superficial de material en $\mathrm{J} / \mathrm{s} * \mathrm{~m}^{2} *{ }^{\circ} \mathrm{C}$

$\mathrm{m}=$ Masa del disco de freno en $\mathrm{Kg}$

$\mathrm{C}_{\mathrm{p}}=$ Calor específico del material en $\mathrm{J} / \mathrm{Kg} *{ }^{\circ} \mathrm{C}$

$\mathrm{t}=$ Tiempo de enfriamiento de Newton en segundos

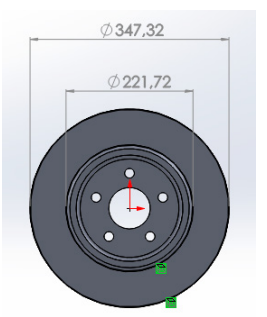

Fig. 2. Diámetro interior y exterior del disco. Fuente: elaboración propia.

Como el disco de freno hace contacto con las pastillas mediante dos superficies, el área de la superficie de contacto aproximada tiene la siguiente ecuación:

$$
\mathrm{A}_{\text {contacto }}=2 * \Pi *\left(\mathrm{r}_{\text {ext }}{ }^{2}-\mathrm{r}_{\text {int }}{ }^{2}\right)=0,1122 \mathrm{~m}^{2}
$$

\begin{tabular}{|c|c|}
\hline \multicolumn{2}{|c|}{ Disco } \\
\hline Tiempo (s) & $\mathrm{Ti}\left({ }^{\circ} \mathbf{C}\right)$ \\
\hline 0 & 60,5 \\
\hline 600 & 45,555 \\
\hline 1200 & 36,411 \\
\hline 1800 & 30,817 \\
\hline 2400 & 27,394 \\
\hline 3000 & 25,300 \\
\hline 3600 & 24,019 \\
\hline 4200 & 23,235 \\
\hline 4800 & 22,755 \\
\hline 5400 & 22,462 \\
\hline 6000 & 22,282 \\
\hline 6600 & 22,173 \\
\hline 7200 & 22,105 \\
\hline 7800 & 22,064 \\
\hline 8400 & 22,039 \\
\hline 9000 & 22,024 \\
\hline 9600 & 22,014 \\
\hline 10200 & 22,009 \\
\hline 10800 & 22,005 \\
\hline 11400 & 22,003 \\
\hline 12000 & 22,002 \\
\hline 12600 & 22,001 \\
\hline 13200 & 22,000 \\
\hline
\end{tabular}

Despejando $T_{i}$ de la ecuación de enfriamiento de Newton, se obtiene la función de tiempo donde se muestra la temperatura del disco a cada instante:

Tabla 2. Temperatura de Enfriamiento de Newton.

Fuente: elaboración propia.

De lo que se obtuvo la siguiente Fig. 3:

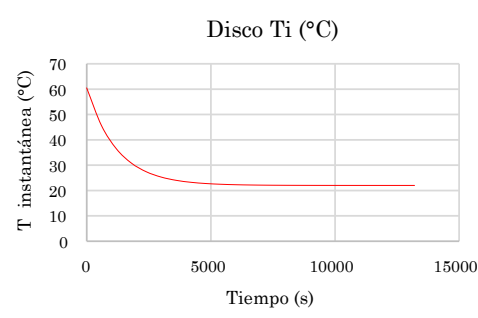

Fig. 3. Enfriamiento de Newton Fuente: elaboración propia.

El disco de freno, luego de haber sido sometido a una temperatura de $60,5^{\circ} \mathrm{C}$ en una frenada, tomará una temperatura ambiente de $22^{\circ} \mathrm{C}$ en aproximadamente 10 minutos, siempre y cuando el enfriamiento se dé por convección natural, fenómeno en el que el aire del ambiente deber tener una velocidad cercana a cero, por otra parte, si el aire estuviera inmóvil, el fenómeno sería solo por conducción. 


\section{Tipos de convección que afectan al disco.}

El disco se ve afectado por varios tipos de convección: en la parte frontal, en sus laterales y en su periferia. A continuación, se determinará la transferencia de calor por convección en cada tipo [38][39].

Convección frontal en el disco. Este tipo de convección afecta la periferia del disco de freno. El flujo de aire sobre un cilindro exhibe patrones complejos, el fluido que se aproxima al cilindro se ramifica y lo rodea, formando una capa límite que lo envuelve (Fig. 4). Las partículas de fluido sobre el plano medio chocan contra el cilindro en el punto de estancamiento y la consecuencia de esto es la elevación de presión en ese punto [29].

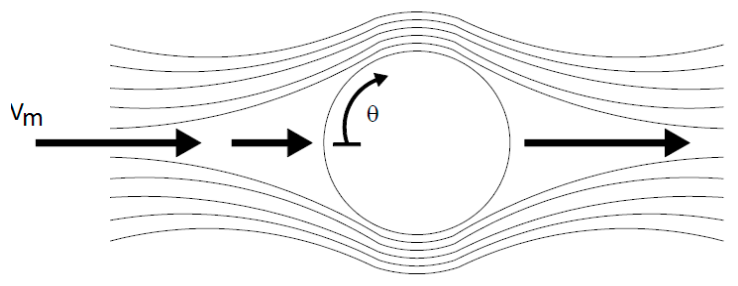

Fig. 4. Flujo alrededor de un cilindro.

$$
\text { Fuente: [37] }
$$

Para el desarrollo de este tipo de transferencia de calor se utilizaron los datos del vehículo que está a una velocidad de $80 \mathrm{Km} / \mathrm{h}$, una temperatura ambiente de $22^{\circ} \mathrm{C}$ y una temperatura superficial de los discos de $60,5^{\circ} \mathrm{C}$, aproximadamente, obtenida en el cálculo de la temperatura superficial al momento del frenado.

Convección periférica. Los cálculos de convección periférica se realizaron con los valores de las propiedades del aire a una temperatura promedio entre el ambiente y la superficie de fricción del disco, en este caso la temperatura superficial, como se muestra en la Fig. 5:

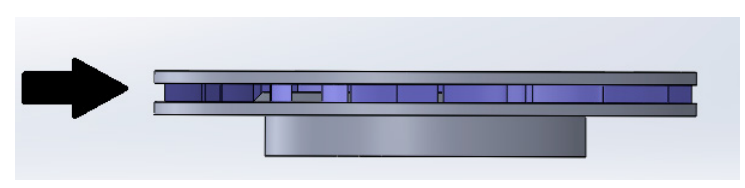

Fig. 5. Convección periférica. Fuente: elaboración propia.

La temperatura periférica se haya mediante la siguiente ecuación:

$$
\mathrm{T}_{\mathrm{f}}=\frac{\mathrm{T}_{\infty}+\mathrm{T}_{1}}{2}=41,25^{\circ} \mathrm{C}
$$

Teniendo la temperatura periférica del aire, se buscarán las propiedades en la Tabla A-17 del libro de transferencia de calor [37].
TABLA 3. PROPIEDADES DEL AIRE A $41,25^{\circ} \mathrm{C}$.

\begin{tabular}{|l|l|}
\hline Número de Prandtl $(\mathrm{Pr})$ & 0,7251 \\
\hline Viscosidad cinemática $\mathrm{v}\left(\mathrm{m}^{2} / \mathrm{s}\right)$ & $1,714^{*} 10^{-5}$ \\
\hline Conductividad térmica $\mathrm{k}\left(\mathrm{W} / \mathrm{m}^{*}{ }^{\circ} \mathrm{C}\right)$ & 0,02671 \\
\hline
\end{tabular}

Fuente: [37]

Para calcular el número de Reynolds, se tiene la siguiente ecuación, que es la de diámetros externos para discos:

$$
\operatorname{Re}=\frac{\mathrm{V}_{\mathrm{m}} * \mathrm{D}}{\mathrm{v}}
$$

Donde $v_{m}$ es la velocidad media del aire, que para este cálculo es de $80 \mathrm{~km} / \mathrm{h}$ y equivale a $22,22 \mathrm{~m} / \mathrm{s}$; el diámetro exterior del disco porque es el área de acción del fluido; y la viscosidad cinemática del aire. Así que se tiene lo siguiente:

$$
\operatorname{Re}=450389,28
$$

El número de Nusselt para este número de Reynolds equivale a:

$$
\mathrm{Nu}=0,027 * \operatorname{Re}^{0,805 * \operatorname{Pr}^{\frac{1}{3}}}=862,91
$$

Con este valor del Nusselt se puede encontrar el coeficiente de convección aplicando la siguiente ecuación:

$$
\mathrm{h}=\frac{\mathrm{k}}{\mathrm{D}} * \mathrm{Nu}
$$

Donde $h$ es el coeficiente de convección que se utilizará para hallar la cantidad de energía disipada en la periferia del disco. Por lo tanto, se tiene:

$$
\mathrm{h}=66,36 \frac{\mathrm{W}}{\mathrm{m}^{2 *}{ }^{\circ} \mathrm{C}}
$$

Ahora se debe calcular el área superficial de la periferia del disco, la cual es:

$$
\mathrm{As}=\Pi * \mathrm{D} * \mathrm{e}
$$

El espesor se muestra en la Fig. 6.

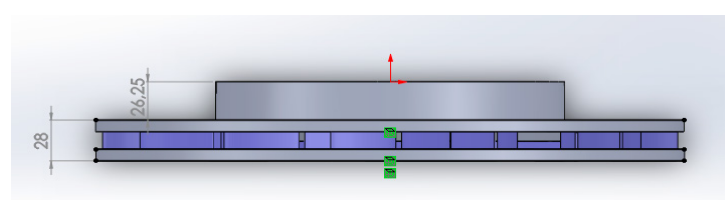

Fig. 6. Espesores del disco. Fuente: elaboración propia.

Por lo tanto, de la ecuación (17), se tiene el área superficial:

$$
\text { As }=\Pi * 0,34732 * 0,028=0,0305 \mathrm{~m}^{2}
$$

Teniendo todos estos valores, se puede encontrar el calor disipado por convección en la periferia.

$$
\mathrm{Q}=\mathrm{h} * \mathrm{As} *\left(\mathrm{~T}_{\mathrm{s}}-\mathrm{T}_{\infty}\right)=78,05 \mathrm{~W}
$$


Convección en la campana. Para calcular el calor disipado en la campana se tienen los mismos datos, solo que varía el diámetro y el espesor, que se muestran en la Fig. 7:

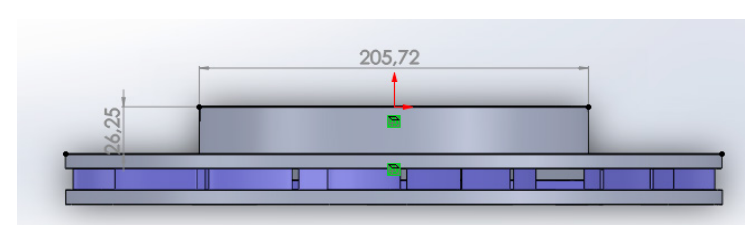

Fig. 7. Espesor y diámetro de la campana del disco. Fuente: elaboración propia.

El número de Reynolds es:

$\operatorname{Re}=\frac{\underline{\mathrm{v}}_{\underline{\mathrm{m}}}}{\mathrm{v}}=266691,85$

El número de Nusselt está dado por:

$\mathrm{Nu}=0,027 * \operatorname{Re}^{0,805} * \operatorname{Pr}^{\frac{1}{3}}=565,93$

El coeficiente de convección será:

$\mathrm{h}=\frac{\mathrm{k}}{\mathrm{D}} * \mathrm{Nu}=73,48 \frac{\mathrm{W}}{\mathrm{m}^{2} *{ }^{\circ} \mathrm{C}}$

Donde:

$\mathrm{k}=$ Conductividad térmica

$\mathrm{D}$ = Diámetro de la campana del disco

$\mathrm{Nu}=$ Número de Nusselt

El área superficial es:

As $=\Pi * \mathrm{D} * \mathrm{e}=0,01696 \mathrm{~m}^{2}$

El calor disipado por convección en la periferia de la campana es:

$\dot{\mathrm{Q}}=\mathrm{h} * \mathrm{As} *\left(\mathrm{~T}_{\mathrm{s}}-\mathrm{T}_{\infty}\right)=48 \mathrm{~W}$

Por lo tanto, el calor total disipado en la periferia del disco es:

$$
\dot{\mathrm{Q}}_{\text {Total }}=48 \mathrm{~W}+78,05 \mathrm{~W}=126,25 \mathrm{~W}
$$

Convección lateral en el disco. No solo en la parte frontal del disco ocurre una transferencia de calor por convección. También en la parte lateral de éste, por lo tanto, se desarrollará el análisis de la pérdida de calor.

Se trabajará como un flujo paralelo en placas planas para los cálculos. Si se supone una placa plana sobre la que fluye una corriente fluida, lo primero que sucede es que la velocidad del fluido disminuye a medida que nos aproximamos hacia la superficie de la misma como consecuencia de las fuerzas de viscosidad. La transmisión de flujo laminar hacia el turbulento depende de la configuración geométrica de la superficie, de su aspereza, de la velocidad de la corriente arriba, de la temperatura superficial y del tipo de fluido, y se caracteriza por el número de Reynolds. El número de Reynolds varía para una placa plana a lo largo del flujo hasta que llegara al final de la placa.
Para el flujo de una capa, suele considerarse que la transmisión de flujo laminar a turbulento ocurre en el número crítico de Reynolds [1][40].

Primero, se debe calcular el número de Reynolds para determinar si es un flujo laminar o turbulento según el número de Reynolds crítico, que es: $\operatorname{Re}_{\mathrm{cr}}=5 * 10^{5}$.

Si el número de Reynolds sobrepasa el valor anterior es turbulento, y si no lo hace, es laminar. Para realizar este cálculo, se supone que el disco es una placa plana cuadrada de longitud $0,34732 \mathrm{~m}$, la temperatura del medio ambiente es de $22^{\circ} \mathrm{C}$, la temperatura superficial del disco de $60,5^{\circ} \mathrm{C}$, con una velocidad del aire de $22,22 \mathrm{~m} / \mathrm{s}$ y a una atmósfera de presión.

La ecuación para el número de Reynolds laminar está dada por:

$$
\mathrm{Re}_{\mathrm{l}}=\frac{\mathrm{v}_{\mathrm{m}} * \mathrm{~L}}{\mathrm{v}_{\mathrm{d}}}
$$

Donde $v_{m}$ es la velocidad media del aire en $\mathrm{m} / \mathrm{s}$, $L$ es la longitud de la placa en $m$, y la viscosidad cinemática de Denver en $\mathrm{m}^{2} / \mathrm{s}$. Las propiedades del aire están en función de la temperatura superficial previamente calculada. La viscosidad de Denver es igual a la viscosidad cinemática, ya que se está trabajando a $1 \mathrm{Atm}$. Por lo tanto, la ecuación queda de la siguiente manera:

$$
\mathrm{Re}_{\mathrm{l}}=450259,6
$$

Esto quiere decir que el flujo es laminar y se usa la siguiente ecuación para determinar el número de Nusselt:

$$
\mathrm{Nu}=400,28
$$

Con el valor del número de Nusselt, se calcula el coeficiente de convección para placas planas con el diámetro mayor del disco, que en este caso será la longitud, según la siguiente ecuación:

$$
\mathrm{h}=\frac{\mathrm{k}}{\mathrm{l}} * \mathrm{Nu}=30,78 \frac{\mathrm{W}}{\mathrm{m}^{2} *{ }^{\circ} \mathrm{C}}
$$

Ya se calculó el área superficial de las dos caras del disco teniendo en cuenta la ecuación (12), que fue:

$$
\mathrm{A}_{\text {contacto }}=0,1122 \mathrm{~m}^{2}
$$

Así que la velocidad de transferencia de calor del disco en las partes laterales del disco está dada por:

$$
\dot{\mathrm{Q}}=\mathrm{h} * \mathrm{As} *\left(\mathrm{~T}_{\mathrm{s}}-\mathrm{T}_{\infty}\right)=132,96 \mathrm{~W}
$$

\section{Resultados y Discusión}

\section{A. Diseño del disco en prueba de agua en el software SolidWorks.}

Se realizó un análisis numérico para estimar la pérdida de calor. Un disco ventilado disipa el calor más rápido que un disco o tambor sólido debido a las diferencias en la geometría. El análisis se realizó en SolidWorks con la biblioteca CFD y con un mallado 
sencillo. El material de freno de disco era de hierro fundido.

Para modelar el flujo de fluido, SolidWorks resuelve las ecuaciones de Navier-Stokes, que son formulaciones de las leyes de conservación de masa, momento y energía [41].

Antes de diseñar el disco de freno completo, lo primero es evaluar el diseño propuesto en un banco de pruebas donde se pueda medir la velocidad a la que el flujo va dentro de la pista del freno [42].

Inicialmente, se diseñó este tipo de disco para la prueba en agua, pero en las pruebas se presentó turbulencia en el centro y las imágenes obtenidas no daban un buen resultado, así que se diseñó otro tipo de disco donde succionara mejor las partículas que, posteriormente, se mostrarán en la fase tres del proyecto. El nuevo diseño en acrílico se presenta en la Fig. 8:

\section{A. Prueba inicial}

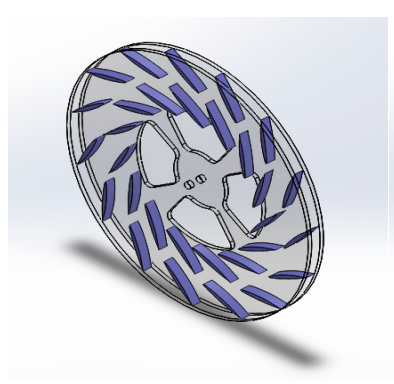

B. Prueba mejorada

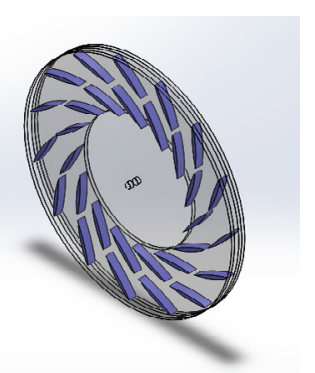

Fig. 8. Ubicación de los pilares tipo NACA. Fuente: elaboración propia.

Con este nuevo diseño se tiene una mejor succión y menos turbulencia en el centro del disco.

A continuación, se realiza un análisis térmico del disco en estado transitorio en el programa ANSYS para determinar la energía disipada y los puntos donde la temperatura es más alta (Fig. 9).

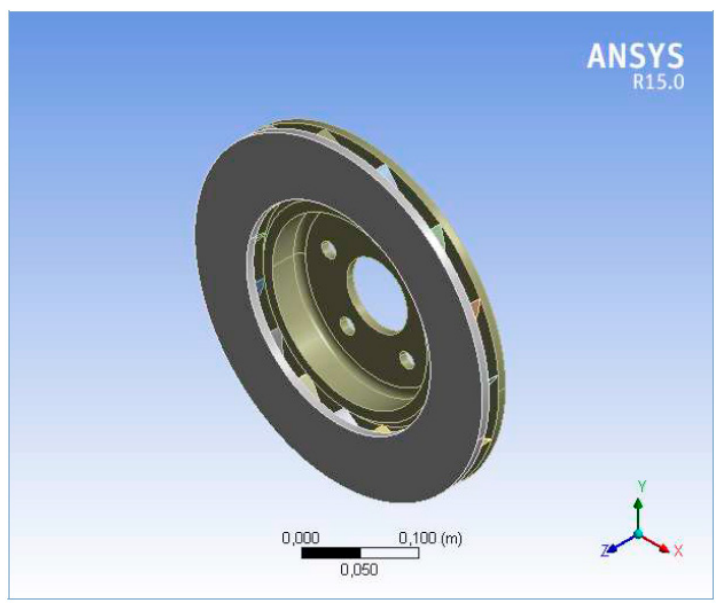

Fig. 9. Freno de disco diseñado en Ansys. Fuente: elaboración propia.
Con los cálculos analizados, se logra obtener una temperatura en la superficie de $67,5^{\circ} \mathrm{C}$ cuando el vehículo va a una velocidad de $22,22 \mathrm{~m} / \mathrm{s}$. Con estos datos iniciales se procede a realizar el análisis en Ansys para determinar la temperatura en el disco al momento de frenar y observar qué sucede al pasar el tiempo, como lo muestra la Fig. 10.

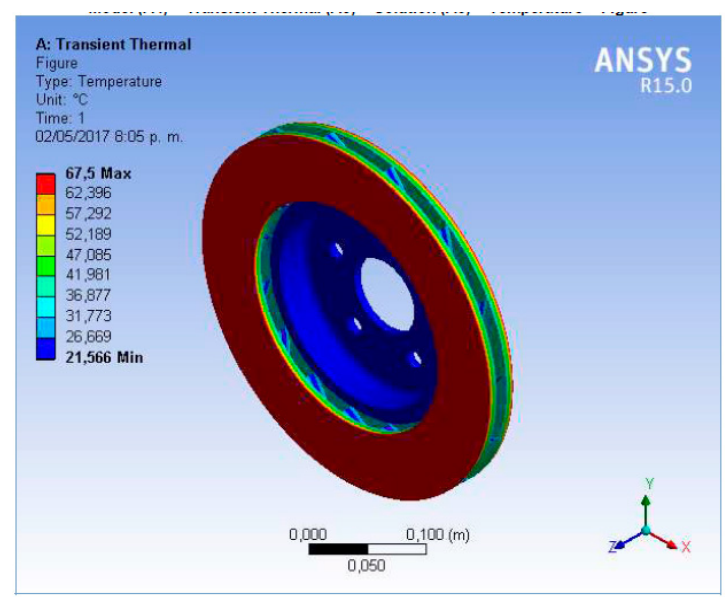

Fig. 10. Análisis térmico transitorio. Fuente: elaboración propia.

En este análisis se puede observar cómo la temperatura va disminuyendo a medida que se acerca a los álabes, y estos no llegan a aumentar su temperatura. Esto quiere decir que el disco tiene una disipación de calor óptima en el interior (rápida en función del tiempo) debido a la geometría de los canales de ventilación.

También se analizó el flujo de calor que se presenta en el disco al transcurrir un segundo, como lo muestra la Fig. 11.

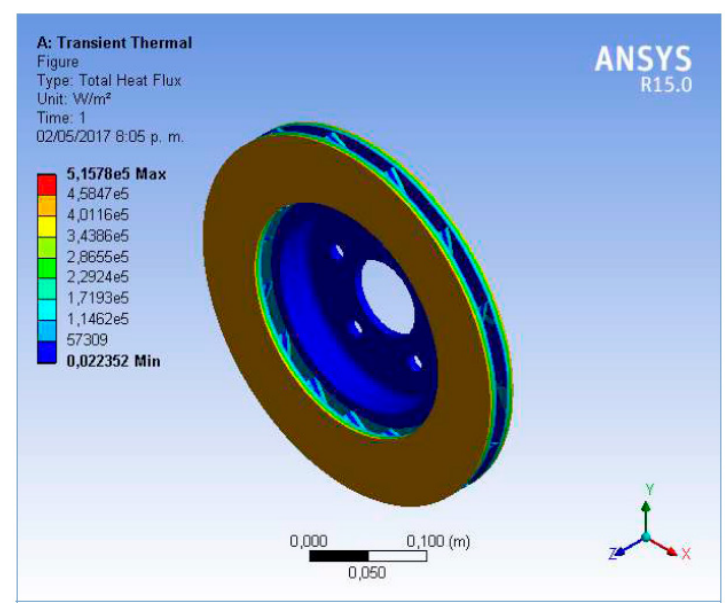

Fig. 11. Análisis de flujo de calor transitorio. Fuente: elaboración propia.

A continuación, en la tabla 4 se muestra el seguimiento del flujo de calor a través del tempo. 
Tabla 4. Datos del Flujo de Calor en el disco en 1 Segundo

\begin{tabular}{|c|c|c|}
\hline Tiempo (s) & Mínimo (w/m²) & Máximo (w/m^2) \\
\hline $1,00 \mathrm{E}-02$ & 0,12682 & $1,97 \mathrm{E}+06$ \\
\hline $2,00 \mathrm{E}-02$ & 0,11895 & $1,87 \mathrm{E}+06$ \\
\hline $5,00 \mathrm{E}-02$ & 0,12669 & $1,63 \mathrm{E}+06$ \\
\hline 0,10394 & $1,97 \mathrm{E}-02$ & $1,34 \mathrm{E}+06$ \\
\hline 0,17348 & 0,12337 & $1,08 \mathrm{E}+06$ \\
\hline 0,26875 & $9,60 \mathrm{E}-02$ & $8,61 \mathrm{E}+05$ \\
\hline 0,36875 & $6,78 \mathrm{E}-02$ & $7,57 \mathrm{E}+05$ \\
\hline 0,46875 & $5,32 \mathrm{E}-02$ & $6,91 \mathrm{E}+05$ \\
\hline 0,56875 & $3,76 \mathrm{E}-02$ & $6,43 \mathrm{E}+05$ \\
\hline 0,66875 & $3,48 \mathrm{E}-02$ & $6,05 \mathrm{E}+05$ \\
\hline 0,76875 & $3,93 \mathrm{E}-02$ & $5,74 \mathrm{E}+05$ \\
\hline 0,86875 & $1,49 \mathrm{E}-02$ & $5,47 \mathrm{E}+05$ \\
\hline 0,93437 & $4,76 \mathrm{E}-02$ & $5,31 \mathrm{E}+05$ \\
\hline 1 & $2,24 \mathrm{E}-02$ & $5,16 \mathrm{E}+05$ \\
\hline
\end{tabular}

Fuente: elaboración propia.

Aquí se puede observar cómo el flujo de calor va disminuyendo de manera considerable en tan solo un segundo, pasando de $1.966 .100 \mathrm{~W} / \mathrm{m}^{2}$ a $515.780 \mathrm{~W} / \mathrm{m}^{2}$. Esto quiere decir que el disco es capaz de disipar el flujo de calor de una manera muy rápida gracias a que la posición de los álabes permite que el fluido (aire) no se concentre en la pista, sino que rápidamente lo evacúe. En la Fig. 12 se puede observar de una mejor manera.

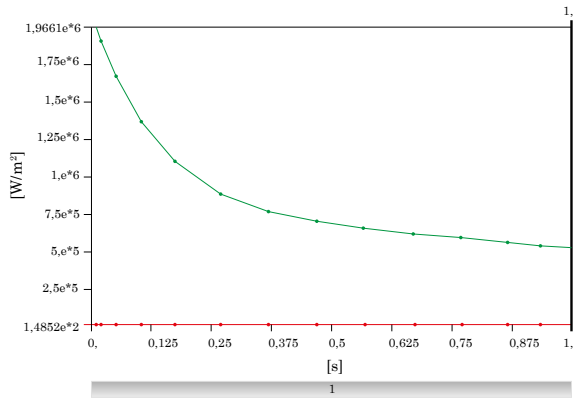

Fig. 12. Flujo de calor en 1 segundo. Fuente: elaboración propia.

\section{Conclusiones}

El estudio del proyecto se basó principalmente en el diseño geométrico, y para que el disco de freno sea implementado en la industria automovilística es necesario estudiar otras dos fases, que son: el tipo de material que tendrá el disco y el proceso de manufactura. Además, se realizó un estudio sobre los materiales que serían implementados en este disco, que son alrededor de quince aleaciones diferentes. De este modo, para que este disco sea implementado en la industria automovilística es necesario estudiar el proceso de manufactura y todos sus requerimientos.
Cabe destacar que el modelo presentado aún es susceptible de modificaciones para mejorar la cantidad de aire que pueda pasar a través de los pilares de ventilación, debido a que otro modelo de pilares o la configuración de estos, así como la cantidad, puede brindar resultados factibles para este estudio. Si se escoge otro perfil de álabes, debe estudiarse el balance entre el arrastre y la sustentación óptimos, además de que la metodología seguida sea la adecuada para poder determinar o cuantificar la velocidad de flujo.

Los cálculos de generación y transferencia de calor se realizaron con base en la velocidad máxima permitida en carretera de $80 \mathrm{Km} / \mathrm{h}$. Por lo tanto, si se quiere emplear velocidades mayores, el disco debe ser sometido a prueba, junto con el material de fabricación, $\mathrm{y}$, de esta forma, tener un estudio completo donde se producirán altas temperaturas y mayor transferencia de calor.

\section{FinANCiAmiento}

Artículo de investigación derivado del proyecto titulado: "Estudio experimental del comportamiento dinámico del flujo de aire a través de un disco de freno automotriz con pilares de ventilación tipo naca 66-209”. Financiado por la Universidad Francisco de Paula Santander, Ocaña. Fecha de inicio: febrero de 2017. Fecha de finalización: julio de 2017.

\section{REFERENCIAS}

[1] R. A. García-León, M. A. Acosta y E. Flórez, "Análisis del comportamiento de los frenos de disco de los vehículos a partir de la aceleración del proceso de corrosión," Tecnura, vol. 19, no. 45, pp. 53-63, 2015. https://doi.org/10.14483/udistrital.jour. tecnura.2015.3.a04

[2] S. Hirasawa, T. Kawanami y K. Shirai, "Numerical analysis of convection heat transfer on high-temperature rotating disk at bottom surface of air flow duct," in ASME International Mechanical Engineering Congress and Exposition, Proceedings (IMECE), vol. 8A, 2014. https://doi.org/10.1115/IMECE2014-36142

[3] A. Ocampo Ramírez, "Diseño de un molino para reciclar pastas de freno de tractocamiones usando el método del elemento finito,» Instituto Politécnico Nacional, 2013.

[4] L. S. Bocîi, "The influence of braking time on heat flow through the friction surfaces of the friction elements of disk brakes for railway vehicles," Transport, vol. 26, no. 1, pp. 75-78, 2011. https://doi.org/10.3846/16484142.2011.563494

[5] H. B. Yan, S. S. Feng, X. H. Yang y T. J. Lu, "Role of cross drilled holes in enhanced cooling of ventilated brake discs," Appl. Therm. Eng., vol. 91, pp. 318-333, 2015. https://doi. org/10.1016/j.applthermaleng.2015.08.042

[6] Z. Chi, Y. He y G. Naterer, "Convective heat transfer optimization of automotive brake discs," SAE Int. J. Passeng. Cars - Mech. Syst., vol. 2, no. 1, pp. 961-969, 2009.

[7] F. Klimenda, J. Soukup y J. Kampo, "Heat distribution in disc brake," in AIP Conference Proceedings, vol. 1745, 2016. https:// doi.org/10.1063/1.4953715

[8] W. Wu, Z. Xiong, J. Hu y S. Yuan, "Application of CFD to model oil-air flow in a grooved two-disc system," Int. J. Heat Mass Transf., vol. 91, pp. 293-301, 2015. https://doi.org/10.1016/j. ijheatmasstransfer.2015.07.092

[9] T. K. R. Rajagopal, R. Ramachandran, M. James y S. C. Gatlewar, "Numerical investigation of fluid flow and heat transfer characteristics on the aerodynamics of ventilated disc brake rotor using CFD," Therm. Sci., vol. 18, no. 2, pp. 667-675, 2014. https://doi.org/10.2298/TSCI111219204R 
[10] Brembo, "The benefits of the new design for the brake disc ventilation chamber," 2015. http://www.brembo.com/en/company/news/new-ventilation

[11] M. N. Dhaubhadel, "CFD applications in the automotive industry (invited keynote presentation)," Am. Soc. Mech. Eng. Fluids Eng. Div. FED, vol. 239, pp. 473-480, 1996.

[12] J. Wurm, M. Fitl, M. Gumpesberger, E. Väisänen y C. Hochenauer, "Novel CFD approach for the thermal analysis of a continuous variable transmission (CVT)," Appl. Therm. Eng., vol. 103, pp. 159-168, 2016. https://doi.org/10.1016/j.applthermaleng.2016.04.092

[13] M. Pevec, I. Potrc, G. Bombek y D. Vranesevic, "Prediction of the cooling factors of a vehicle brake disc and its influence on the results of a thermal numerical simulation," Int. J. Automot. Technol., vol. 13, no. 5, pp. 725-733, 2012. https://doi. org/10.1007/s12239-012-0071-y

[14] D. Wei, J. Ruan, W. Zhu y Z. Kang, "Properties of stability, bifurcation, and chaos of the tangential motion disk brake," J. Sound Vib., vol. 375, pp. 353-365, 2016. https://doi. org/10.1016/j.jsv.2016.04.022

[15] J. Ruan, P. D. Walker, P. A. Watterson y N. Zhang, "The dynamic performance and economic benefit of a blended braking system in a multi-speed battery electric vehicle," Appl. Energy, vol. 183, pp. 1240-1258, 2016. https://doi.org/10.1016/j. apenergy.2016.09.057

[16] M. Gulec, E. Yolacan y M. Aydin, "Design, analysis and real time dynamic torque control of single-rotor-single-stator axial flux eddy current brake," IET Electr. Power Appl., vol. 10, no. 9, pp. 869-876, 2016. https://doi.org/10.1049/iet-epa.2016.0022

[17] W. Wei, Y. Hu, Q. Wu, X. Zhao, J. Zhang y Y. Zhang, "An air brake model for longitudinal train dynamics studies," Vehicle System Dynamics. Taylor and Francis Ltd., School of Traffic and Transportation Engineering, Dalian Jiaotong University, Dalian, People's Republic of China, pp. 1-17, 2016.

[18] A. Shahril, R. Samin, J. M. Juraidi y J. Daut, "Structural analysis of brake disc using dynamic simulation," ARPN J. Eng. Appl. Sci., vol. 10, no. 17, pp. 7805-7808, 2015.

[19] U. Andreaus y P. Casini, "Dynamics of friction oscillators excited by a moving base and/or driving force," J. Sound Vib., vol. 245, no. 4, pp. 685-699, 2001. https://doi.org/10.1006/ jsvi.2000.3555

[20] S. W. Shaw, "On the dynamic response of a system with dry friction," J. Sound Vib., vol. 108, no. 2, pp. 305-325, Jul. 1986. https://doi.org/10.1016/S0022-460X(86)80058-X

[21] D. Meng, L. Zhang y Z. Yu, "A dynamic model for brake pedal feel analysis in passenger cars," Proc. Inst. Mech. Eng. Part D J. Automob. Eng., vol. 230, no. 7, pp. 955-968, 2016. https:// doi.org/10.1177/0954407015598030

[22] S.-D. Lee y S.-L. Kim, "Characterization and development of the ideal pedal force, pedal travel, and response time in the brake system for the translation of the voice of the customer to engineering specifications," Proc. Inst. Mech. Eng. Part D J. Automob. Eng., vol. 224, no. 11, pp. 1433-1450, 2010. https:// doi.org/10.1243/09544070JAUTO1585

[23] V. Surblys y E. Sokolovskij, "Research of the Vehicle Brake Testing Efficiency," in 9th International Scientific Conference, Transbaltica, vol. 134, pp. 452-458, 2015, 2016. https://doi. org/10.1016/j.proeng.2016.01.067

[24] A. Q. Xu, "Study on the dynamic characteristics of a high frequency brake based on giant magnetostrictive material," Smart Mater. Struct., vol. 25, no. 6, 2016. https://doi. org/10.1088/0964-1726/25/6/065001

[25] E. Palmer, R. Mishra y J. Fieldhouse, "An optimization study of a multiple-row pin-vented brake disc to promote brake cooling using computational fluid dynamics," Proc. Inst. Mech. Eng. Part D J. Automob. Eng., vol. 223, no. 7, pp. 865-875, 2009. https://doi.org/10.1243/09544070JAUTO1053

[26] A. Nagarajan y M. R. Narayanan, "Maximization of efficiency for Disk brake material using composite material by modelling and analysis," Int. J. Control Theory Appl., vol. 9, no. 6, pp. 2793-2798, 2016.

[27] R. A. García-León y E. Perez Rojas, "Analysis of the amount of heat flow between cooling channels in three vented brake discs," Ing. y Univ., vol. 21, no. 1, pp. 55-70, 2017.

[28] L. Pan, J. Han, Z. Li, Z. Yang y W. Li, "Numerical simulation for train brake disc ventilation," Beijing Jiaotong Daxue Xuebao/Journal Beijing Jiaotong Univ., vol. 39, no. 1, pp. 118-124, 2015 .
[29] Y. Cengel, Tansferencia de calor y masa. Un enfoque práctico. Tercera edición. México: McGraw-Hil, 2007.

[30] D. R. Flores Galindo, "Diseño de perfiles aerodinámicos,» Instituto Politécnico Nacional, 2006.

[31] Airfoill Tools, «NACA 66-209 (naca66209-il),» 2017.

[32] UIUC, "Applied Aerodynamics Group," University of Illinois, 2017. [Online]. Available: http://m-selig.ae.illinois.edu/ads/ coord_database.html\#N.

[33] R. A. García-León, "Evaluación del comportamiento de los frenos de disco de los vehículos a partir del análisis de la aceleración del proceso de corrosión," Universidad Francisco de Paula Santander Ocaña, 2014.

[34] R. Limpert, Brake Design and Safety, 2a ed. Society of Auto motive Engineers, 1999.

[35] A. Cruz Azanza, C. A. Jiménez García y M. Á. Ordaz Flores, "Diseño geométrico de un prototipo optimizado de un disco de freno automotriz ventilado,» 2012 .

[36] M. U. Ramírez, "Instituto politécnico nacional,» 2012

[37] Y. A. Cengel y M. E. Boles, «Termodinámica - Cengel 7th,» Termodinámica, p. 1456, 2011.

[38] R.A. García-León, "Thermal study in three vented brake discs, using the finite element analysis," DYNA, vol. 84, no. 200, pp. 19-27, 2017. https://doi.org/10.15446/dyna.v84n200.55663

[39] R. A. García-León y E. Flórez-Solano, "Dynamic analysis of three autoventilated disc brakes," Ing. e Investig., vol. 37, no. 3, pp. 102-114, 2017. https://doi.org/10.15446/ing.investig. v37n3.63381

[40] R. A. García-León y E. Flórez, "Estudio analítico de la transferencia de calor por convección que afectan los frenos de disco ventilados," Tecnura, vol. 20, pp. 15-30, 2016.

[41] R. A. García León y E. Pérez Rojas, "Analysis of the amount of heat flow between cooling channels in three vented brake discs," Ing. y Univ., vol. 21, no. 1, pp. 71-96, 2017.

[42] D. Porta, C. Echeverría, A. Aguayo, J. E. H. Cardoso y C. Stern, Recent Advances in Fluid Dynamics with Environmental Applications, 2016.

Ricardo Andrés García-León. Ingeniero mecánico de la Universidad Francisco de Paula Santander de Ocaña en 2014. Magíster en Ingeniería Industrial de la Universidad de Pamplona, Colombia. Estudiante de doctorado en Ingeniería Mecánica del Instituto Politécnico Nacional de México. Vinculado desde el 2014 como coordinador del grupo de investigación INGAP y en el 2015 como docente catedrático del Departamento de Ingeniería Mecánica de la Facultad de Ingenierías de la Universidad Francisco de Paula Santander, Ocaña. Investigador y coordinador de la línea de investigación Materiales y Procesos Industriales. Sus áreas de interés son principalmente el desarrollo de sistemas mecánicos, procesos industriales y materiales de ingeniería. https://orcid.org/0000-0002-2734-1425

Robert Dajjan Echavez-Díaz. Ingeniero mecánico de la Universidad Francisco de Paula Santander de Ocaña en 2017. Estudiante de la maestría en Ingeniería de Manufactura del Instituto Politécnico Nacional de México. Perteneció al semillero de investigación DETIMEC hasta el 2017. Adscrito al grupo de investigación INGAP. https://orcid.org/0000-0002-6098-0923

Eder Flórez-Solano. Ingeniero mecánico de la Universidad Francisco de Paula Santander. Magíster en Ingeniería Mecánica de la Universidad del Táchira, Venezuela. Estudiante de doctorado en Ingeniería Mecánica. Vinculado desde el 2009 como docente de tiempo completo y director del grupo de investigación INGAP. Su áreas de interés es principalmente el desarrollo de sistemas mecánicos. https://orcid.org/00000003-2527-0413 